\title{
AUTOMATIZAÇÃO DE PULVERIZADOR PNEUMÁTICO TRATORIZADO EM FUNÇÃO DO DÉFICIT DE PRESSÃO DE VAPOR D’ÁGUA NO AR
}

\author{
Gustavo Guethi Manhani ${ }^{1}$, Mauri Martins Teixeira ${ }^{1}$, Haroldo Carlos Fernandes ${ }^{1}$, Sergio Zolnier ${ }^{1}$, \\ Robson Shigueaki Sasaki ${ }^{2}$, Cleyton Batista Alvarenga ${ }^{1}$ \\ ${ }^{1}$ Universidade Federal de Viçosa, ${ }^{2}$ Instituto Federal Minas Gerais, E-mail: guvic03@yahoo.com.br, mauri@ufv.br, \\ haroldo@ufv.br, zolnier@ufv.br, robsonsasaki@yahoo.com.br, cleytonbatista@yahoo.com.br
}

\section{RESUMO}

Objetivou-se com este trabalho desenvolver um sistema automático de controle da vazão de ar de um pulverizador pneumático Berthoud, modelo AF 427, de acordo com as condições meteorológicas no momento da pulverização. Desenvolveu-se um diafragma automatizado posicionado na entrada de ar do ventilador, e avaliou-se a eficiência desse sistema quanto à precisão e tempo de resposta às diferentes condições meteorológicas. O sistema é composto por uma estrutura eletromecânica, sensor de umidade e temperatura, um microcontrolador e um algoritmo para controlar as tarefas de acordo com as informações obtidas pelos sensores. Através do sistema eletromecânico e com o auxílio de sensores de posição e LEDs, coube ao sistema eletrônico posicionar o diafragma adequando a vazão de ar do ventilador conforme a condição meteorológica. Ao final verificou-se que o sistema respondeu à variação das condições meteorológicas. O tempo de resposta do sistema variou de 39,3 a 66,3 segundos, apresentando alta precisão.

Palavras-chave: Fluxo de ar, máquinas agrícolas, evaporação

\section{AUTOMATION OF A CANNON SPRAYER ACCORDING THE WEATHER CONDITIONS AT THE MOMENT OF THE SPRAYING}

\section{ABSTRACT}

The objective of this paper was to develop an automatic system to control air flow rate of a Berthoud cannon sprayer, model AF 427, according to the weather conditionsat the time of spraying. Used an automated diaphragm placed in the air inlet of the fan, and evaluate the efficiency of this system for accuracy and response time to the different weather conditions. The system was basically composed of an electromechanical structure, humidity and temperature sensor, microcontroller and an algorithm to interpret the weather information. With the electromechanical system and with aid of position sensors and LEDs, the electronic system 
positions the diaphragm to adjust the air flow rate of the fan at a given weather condition. At the end it was found that the system responded to changes in weather conditions. The response time of the system varied from 39.3 to 66.3 seconds with high precision.

Keywords: Air flow, agricultural machinery, evaporation

\section{INTRODUÇÃO}

O cultivo de áreas agrícolas remete ao desafio da utilização de agrotóxicos, em consonância com a preservação do meio ambiente, saúde do operador, produção de alimentos sem resíduos e buscando a sustentabilidade do sistema agrícola.

Em uma pulverização, diversos fatores podem ocasionar perdas para o meio ambiente e redução da eficiência da operação. Segundo Villalba \& Hetz (2010), uma combinação de temperatura acima de 30 ${ }^{\circ} \mathrm{C}$ e umidade relativa abaixo de $40 \%$ têm uma forte influência sobre a evaporação das gotas pulverizadas, em especial quando estas são finas. Ruedell (2002) mencionou que a pulverização em condições onde a temperatura do ar for inferior a $10{ }^{\circ} \mathrm{C}$ e superiora $35^{\circ} \mathrm{C}$ deve ser interrompida, sendo que, valores entre 20 e $30{ }^{\circ} \mathrm{C}$ compreendem a faixa ideal para pulverizações. Além disso, $\mathrm{Yu}$ et al. (2009) concluíram que, com a diminuição da umidade relativa de 90 para $30 \%$, o tempo de evaporação de uma gota com diâmetro de $343 \mu \mathrm{m}$ reduziu de 115 para 52 segundos. Em outro trabalho $\mathrm{Yu}$ et al. (2009b) observaram que o tempo de evaporação de gotas com diâmetro de 246 $\mu \mathrm{m}$ em folhas pilosas, foi de $12 \mathrm{~s}$ quando a umidade relativa foi de $30 \%$, e 40 s quando se alterou a umidade para $90 \%$.

Visando obter uma aplicação eficiente, em condições de campo, deve-se atentar aos fatores meteorológicos durante as pulverizações, entretanto, nem sempre é possível pulverizar somente em condições ideais de temperatura e umidade relativa, visto que as condições meteorológicas são incontroláveis e variáveis ao longo do tempo e a janela de aplicação as vezes é reduzida. Neste sentido, pesquisas como as realizadas por Batte \& Ehsani (2006), Doruchowsky et al. (2009) e Balsariet al. (2009) têm focado na precisão e automação de pulverizadores com o intuito de aumentar a eficiência da pulverização e reduzir as perdas.

Doruchowski et al. (2009), utilizaram um pulverizador hidropneumático, dotado de um diafragma automatizado na entrada do ventilador, e verificaram que com o fechamento do diafragma, ocorreu redução da velocidade do ar. A automação do pulverizador apresentou o desempenho 
pretendido com habilidade de alterar os parâmetros da pulverização em tempo real. Em outro trabalho, Alvarenga et al. (2013) desenvolveram um sistema automático para controle do espectro de gotas em um pulverizador hidropneumático em função do déficit de pressão de saturação de vapor d'água no ar. Ao final, observaram que o sistema desenvolvido foi eficiente na manutenção do espectro de gotas durante a pulverização e facilitou as operações de campo, uma vez que é retirada a responsabilidade do operador quanto a observação dos fatores meteorológicos.

Em pulverizadores hidráulicos, já existem no mercado mecanismos dotados de sistema de comutação de pontas de pulverização, o qual possibilita a alteração do espectro de gotas, conforme as condições no momento da aplicação e o tratamento a ser realizado.

Entretanto, na pulverização pneumática, se desconhece a existência de um sistema automatizado que altere o espectro de gotas, sendo que nesse tipo de equipamento o diâmetro das gotas é proporcional à vazão de ar do pulverizador.

Sendo assim objetivou-se desenvolver um sistema automático para o controle da vazão de ar de pulverizador pneumático, conforme as condições meteorológicas, através do controle da abertura de um diafragma e avaliar esse sistema quanto à sua vazão de ar, precisão e tempo de resposta, em diferentes condições meteorológicas.

\section{MATERIAL E MÉTODOS}

O experimento foi realizado nas dependências do Laboratório de Mecanização Agrícola, no Departamento de Engenharia Agrícola da Universidade Federal de Viçosa, Viçosa em Minas Gerais.

Utilizou-se um pulverizador pneumático, marca Berthoud, modelo AF 427, equipado com ventilador radial, acoplado a um trator John Deere, modelo 5705 , e potência nominal de $62,5 \mathrm{~kW}$.

Para o controle da vazão de ar foi construído um diafragma com chapas de aço galvanizadas, fixado na entrada de ar do ventilador do pulverizador. Após a instalação, avaliaram-se as vazões de ar produzidas pelo ventilador, variando-se a abertura do diafragma manualmente. Para determinar a vazão de ar fornecida pelo ventilador, foi utilizado o método estabelecido pela "Air Movingand Conditions Association (AMCA)". Por este método, foi construído um túnel de vento dotado de um tubo homogeneizador de fluxo de ar e de uma válvula cônica controladora 
da vazão na saída do duto. Neste ensaio o pulverizador foi acoplado a um trator que operou com rotação de 219,91 $\operatorname{rad~s}^{-1}$ (2.100 rpm) no conta-giros, produzindo $56,55 \mathrm{rad} \mathrm{s}^{-}$ 1 (540 rpm) na Tomada de Potência (TDP), que correspondeu a uma rotação média de 408,42 $\mathrm{rad} \mathrm{s}^{-1}$ (3.900 rpm) no eixo do ventilador.

Depois de determinadas às vazões de ar, manualmente, prosseguiu-se para a automatização do sistema. Para o controle da abertura do diafragma optou-se pela montagem de uma estrutura eletromecânica que proporcionasse a abertura desejada mediante a atuação de um motor elétrico.

O mecanismo do atuador constituiu-se de um motor elétrico tipo CEP de 12 volts, com consumo nominal de 7,5 A, torque de 25 a $48 \mathrm{Nm}$ e rotação máxima de 100 rpm, acoplado a um parafuso de potência fixado ao pulverizador através de uma estrutura metálica. Este parafuso acionava uma porca acoplada a uma biela de ferro que promoveu a regulagem do diafragma.

Para a leitura das condições do ambiente utilizou-se um sensor de umidade relativa e temperatura do ar INTERCAP $\mathbb{R}$ HMP50, um microcontrolador Arduino Duemilanove para processar os dados provenientes do sensor, juntamente com uma placa de relés, três sensores de posição e um conjunto de três leds, para auxiliar a equipe de pesquisa que acompanhava o trabalho, e o operador na visualização do funcionamento do sistema.

O sensor envia um sinal de tensão ao microcontrolador e este interpreta o sinal como sendo representativo de uma determinada temperatura e umidade relativa do ar (Equações 1 e 2):

$$
\begin{aligned}
& \text { Tar }=-40+100(5 / 1023) \text { Sinal_Tar }(1) \\
& U R=100(5 / 1023) \text { Sinal_UR (2) }
\end{aligned}
$$

Em que:

Tar = temperatura do ar, $\left({ }^{\circ} \mathrm{C}\right) ; \mathrm{e}$, $\mathrm{UR}=$ umidade relativa do ar, (\%).

Um algoritmo foi elaborado para interpretar as informações de temperatura e umidade relativa do ar detectado pelos sensores e, consequentemente, determinar o déficit de pressão.

Através do sistema eletromecânico desenvolvido foi possível regular, automaticamente, o diafragma adequando a vazão de ar do ventilador.

Os déficits de pressão foram previamente estabelecidos de tal forma que valores baixos significam condições ideais para se realizar uma pulverização, podendose trabalhar com gotas de diâmetros reduzidos, neste caso com a abertura total do diafragma. Já quando a condição meteorológica se torna desfavorável, 
necessita-se trabalhar com gotas de diâmetros maiores, necessitando-se assim menor vazão de ar, fechando-se o diafragma, quando o DPV ultrapassou $30,00 \mathrm{hPa}$ a pulverização foi interrompida, sendo o operador orientado a não aplicar nessas condições (Tabela 1).

Após a automatização do sistema, procedeu-se à validação do mesmo quanto ao tempo de resposta do posicionamento do diafragma, e a precisão da resposta do comando. O tempo de resposta foi estudado no intuito de se verificar a velocidade com que o sistema eletrônico e eletromecânico atua sobre o posicionamento do diafragma. Deste modo, sendo possível, estimar no campo a distância percorrida até que o atuador possa abrir ou fechar o diafragma.

Os déficits de pressão de saturação estudados foram obtidos através de aquecimento do ambiente próximo ao sensor através do uso de um aquecedor. Com o estabelecimento das condições meteorológicas desejadas, dava-se início a movimentação do diafragma.

Os componentes do tempo de resposta estudados foram: (1) tempo necessário para movimentar o diafragma da abertura de 100 até $70 \%$; (2) tempo para movimentar o diafragma da abertura de 70 até 50\%; (3) tempo para movimentar o diafragma da abertura de 50 até $70 \%$ e (4) tempo necessário para movimentar o diafragma da abertura de 70 até $100 \%$. Foram realizadas dez repetições para cada coleta dados dos componentes avaliados. Os resultados foram analisados através de estatística descritiva.

Para avaliação da resposta dos sistemas envolvidos em posicionar o diafragma, de acordo com o estabelecido para cada déficit de pressão de vapor d'água no ar, foram realizadas novas medições da vazão de ar do ventilador e, comparadas aos valores obtidos com ajuste manual de posição do diafragma. Procurou-se verificar a precisão do posicionamento do diafragma através dos resultados de vazão de ar coletados.

O experimento foi montado no delineamento inteiramente casualizado onde as vazões de ar foram estudadas para cada intervalo de déficit de pressão de vapor d'água no ar, em dez repetições. Os fatores de estudos foram o tempo de resposta e a homogeneidade do fluxo de ar.

Os resultados de vazão de ar obtidos em cada déficit de pressão foram submetidos à análise de variância e aplicação de teste Tukey a 5\% de probabilidade, para verificação da diferença estatística entre as médias dos tratamentos. 
Tabela 1. Posição de abertura do diafragma em função do déficit de pressão de vapor d'água no ar.

\begin{tabular}{cc}
\hline Abertura do diafragma (\%) & Déficit de pressão de saturação (hPa) \\
\hline 100 & $\mathrm{DPV} \leq 7,01$ \\
70 & $7,01<\mathrm{DPV} \leq 12,67$ \\
50 & $12,67<\mathrm{DPV} \leq 30,00$ \\
Não aplicar & $\mathrm{DPV}>30,00$ \\
\hline
\end{tabular}

\section{RESULTADOS E DISCUSSÃO}

Nos resultados obtidos no ensaio de vazão, antes da automatização do sistema, verificou-se que, quando o diafragma permaneceu totalmente aberto, a vazão de ar foi de $51,28 \mathrm{~m}^{3} \mathrm{~h}^{-1}$, e para a abertura de $70 \%$ e $50 \%$, as vazões de ar foram de 48,94 e $38,97 \mathrm{~m}^{3} \mathrm{~h}^{-1}$, respectivamente, promovendo redução de 4,56 e 24\%, na vazão de ar comparado ao diafragma totalmente aberto.

O funcionamento da estrutura baseouse na rotação do parafuso de potência, com ajuda de um motor elétrico, para girar uma porca em torno do eixo do parafuso. Essa porca promoveu a abertura e o fechamento do diafragma, pela alteração do sentido de rotação do eixo do motor.

O sistema eletrônico foi elaborado com a aquisição de dados realizada via comunicação USB com um "laptop".

A placa controladora de relés faz a interface entre o microcontrolador e $\mathrm{o}$ atuador que acionou o diafragma. Essa placa foi a responsável pela alteração da polaridade da ligação do motor na fonte, alterando o sentido de rotação do motor e, consequentemente, possibilitando o movimento do diafragma.

Os sensores de posição foram adaptados na estrutura eletromecânica nos locais de abertura do diafragma e conectados ao microcontrolador através do sistema "plugand play". Sua função apenas identificar o ponto de parada do parafuso de potência, para obter precisão no funcionamento dos mecanismos.

$\mathrm{O}$ algoritmo identifica a seleção do posicionamento do diafragma e determina a interrupção da aplicação ou alteração necessária conforme a limitação imposta por um déficit de pressão e o operador executa o comando. Esse, por sua vez, teve como uma de suas funções, interpretar a informação da condição em tempo real através de um sistema de controle de LEDs que estão conectados ao microcontrolador. Este sistema de controle foi composto por LEDs 
de cores diferentes, verde, amarelo e vermelho, cada um com sua ação (Figura 1).

O algoritmo continha a lógica de programação relativa aos comandos para cada movimento do diafragma e direciona aos LEDs a via de interpretação para estes movimentos, isto é: o LED verde pisca por dez segundos quando o sistema é inicializado e permanece ligado caso o diafragma esteja na posição correta, simultaneamente informando ao operador que o sistema eletrônico está em constante processo de monitoramento das condições meteorológicas. Caso o diafragma se encontra em uma posição não conhecida, ele é automaticamente movido ao ponto inicial (máxima abertura), porém, não sendo essa a posição desejada, o microcontrolador interpreta a informação e a aciona o atuador que, por sua vez, move o diafragma para a abertura correta.

Quando o déficit de pressão alterou-se e o diafragma estava prestes a se movimentar para uma nova posição, o LED verde era desligado e o LED amarelo piscava por dez segundos, como contagem regressiva do movimento do diafragma que estava para acontecer. No início do movimento o LED amarelo permanecia ligado informando que o diafragma estava deslocando-se para uma nova posição. Uma vez o diafragma atingindo esta posição, o LED amarelo era desligado e o LED verde era novamente conectado, aparecendo a luz verde, indicando que o diafragma atingiu a abertura desejada e o monitoramento contínuo era novamente inicializado.

Em condições meteorológicas desfavoráveis, quando o déficit de pressão estava acima de $30 \mathrm{hPa}$, o LED vermelho era acionado, indicando ao operador que a aplicação deveria ser interrompida. O LED vermelho continuava aceso monitorando as condições do ambiente, até que uma nova ordem de posicionamento fosse enviada pelo microcontrolador.

$\mathrm{Na}$ ocorrência de déficit de pressão intermediária, ocorre a movimentação do diafragma e alteração do espectro de gotas.

Entretanto, em déficit extremamente elevado, o LED vermelho indica interrupção da pulverização, visto que nestas condições, as gotas sofrem forte influência das condições climáticas. 


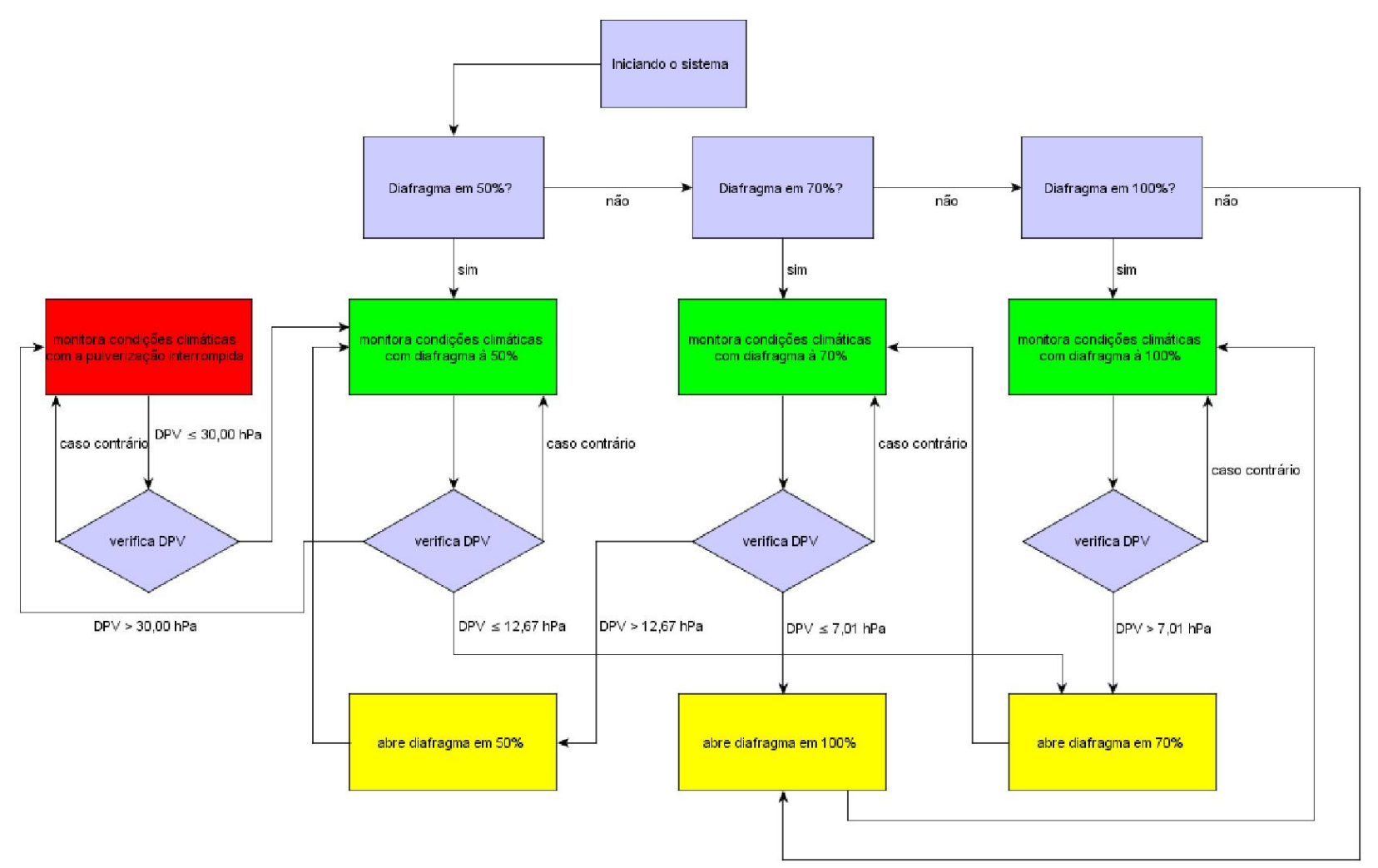

Figura 1. Diagrama de funcionamento do algoritmo.

Alvarenga et al. (2013) avaliaram a influência do déficit de pressão de vapor d'água no ar nos parâmetros da pulverização na cultura da laranja. Observaram redução do diâmetro das gotas em condições extremas de déficit de pressão de vapor d'água no ar e ainda, constataram redução na deposição de líquido no alvo sob estas condições. Sob baixo DPV $(<10 \mathrm{hPa})$, a deposição média foi de $2,6 \mu \mathrm{L} \mathrm{cm}^{-2}$; reduzido para 1,2 e $1,0 \mu \mathrm{L} \mathrm{cm}^{-2}$, quando se aumentou o valor de DPV para 14,5 e $21 \mathrm{hPa}$ respectivamente.

Observa-se que o sistema automatizado desenvolvido, retira a responsabilidade do aplicador, opiniões semelhantes foram expressas por Alvarenga et al. (2013). Cabe ao operador apenas interpretar o conjunto de leds, em que as suas colorações já são interativas e facilmente interpretadas. Com a alteração do diafragma, ocorre redução da vazão de ar do ventilador, logo altera o espectro de gotas pulverizadas (MANHANI et al., 2013). Ou seja, o espectro é controlada em função das condições meteorológicas locais no momento da pulverização. Debortoli et al. (2012) relatam que o espectro de gotas deve ter diâmetro mediano volumétrico suficiente 
para depositar no alvo, sem evaporar, a calda de pulverização com o produto

$\mathrm{Na}$ avaliação do tempo de resposta do sistema, de acordo com a aquisição de dados via USB, o tempo de resposta foi calculado pela diferença entre os horários em que o microcontrolador recebia o sinal para o reposicionamento do diafragma, indicado pelos comandos, abrindo ou fechando o diafragma, e o momento que o sistema retomava o monitoramento contínuo após ter atingido a abertura desejada. O tempo de resposta para o posicionamento do diafragma variou entre 39,3 a 66,3 segundos, com coeficiente de variação máximo de 4,81 \%. Os valores de coeficiente de variação apresentam-se todos abaixo de 5\% representando baixa variabilidade e boa precisão do sistema eletromecânico no posicionamento automático do diafragma (Tabela 2).

Observou-se que os maiores tempos de resposta médios ocorreram no momento do fechamento do diafragma.

Este fato é explicado devido ao sentido de rotação do eixo do motor, que operou na rotação otimizada de trabalho. Isto ocorreu, porque posteriormente ao projeto do diafragma, constatou-se maior atrito entre as partes quando o mesmo era fechado. Por isso, maiores valores de tempo de resposta foram encontrados para este sentido de movimento. Schuller (1991), afirma que o tempo de resposta é um dos fatores-chave para o desempenho das máquinas de aplicação dotadas de controle eletrônico.

Tabela 2. Tempo de resposta do posicionamento do diafragma, Viçosa - MG, julho de 2011.

\begin{tabular}{lcccc}
\hline \multirow{2}{*}{ Posicionamento do diafragma $(\%)$} & \multicolumn{5}{c}{ Tempo de resposta (s) } \\
\cline { 2 - 6 } & $\mathrm{N}$ & Média & Desvio padrão & Coeficiente de variação (\%) \\
\hline 100 para 70 & 10 & 66,3 & 1,77 & 2,67 \\
70 para 50 & 10 & 53,4 & 1,84 & 3,44 \\
50 para 70 & 10 & 39,3 & 1,89 & 4,81 \\
70 para 100 & 10 & 62,0 & 1,83 & 2,94
\end{tabular}

N: Número de observações. 
O maior valor de tempo de resposta médio foi de 66,3 segundos ocorrendo no momento de fechamento do diafragma de 100 para $70 \%$. Considerando o conjunto trator-pulverizador operando a $2,6 \mathrm{~km} \mathrm{~h}^{-1}$, isto proporcionaria um deslocamento de 47,88 metros. Isto demonstra a importância do operador para a atenção nas informações representativas dos LEDs, evitando que ocorram faixas de aplicação com espectro de gotas não recomendado.

Posteriormente a avaliação do tempo de resposta, prosseguiu-se para a avaliação da precisão do sistema, comparando as vazões de ar do sistema manual e automatizado. As condições meteorológicas durante este ensaio, permaneceram com déficit de pressão de saturação de vapor d'água no ar (DPV), identificado pelo sensor durante as tomadas de vazão de ar, de 10,39 $\mathrm{hPa}$, estando na faixa pré-determinada de 7,01 a 12,67 hPa. Observou-se que as médias de vazões de ar, obtidas após o posicionamento automático do diafragma, de acordo com o DPV, apresentam valores próximos em relação às médias obtidas manualmente. Também o pulverizador automatizado foi capaz de produzir vazões de ar estatisticamente diferentes, o que era o propósito do projeto (Tabela 3).

O sistema eletromecânico respondeu, positivamente, para obtenção da vazão de ar estabelecida para uma determinada condição meteorológica. Para todos os intervalos de DPV, verificam-se valores de coeficiente de variação abaixo de 5\%, o que evidencia a precisão do sistema em obter a vazão de ar previamente estabelecida.

A menor vazão de ar proporcionada pelo pulverizador automatizado na condição meteorológica mais crítica responde positivamente o objetivo proposto, uma vez que nessas condições é necessário um menor volume de ar, e por consequência, um menor fracionamento das gotas.

Tabela 3. Vazão de ar do pulverizador automatizado, Viçosa - MG, julho de 2011. Vazão de $\operatorname{ar}\left(\mathrm{m}^{3} \cdot \mathrm{min}^{-1}\right)$

\begin{tabular}{ccccc} 
Intervalo de déficit de pressão $(\mathrm{hPa})$ & & & & \\
\cline { 2 - 5 } & $\mathrm{N}$ & Média* $^{*}$ & Desvio padrão & Coeficiente de variação (\%) \\
\hline $\mathrm{DPV} \leq 7,01$ & 10 & $52,3 \mathrm{a}$ & 1,79 & 3,43 \\
$7,01<\mathrm{DPV} \leq 12,67$ & 10 & $47,7 \mathrm{~b}$ & 0,79 & 1,66 \\
$12,67<\mathrm{DPV} \leq 30,00$ & 10 & $38,5 \mathrm{c}$ & 1,34 & 3,48
\end{tabular}

N: Número de observações.

*Médias seguidas por letras diferentes diferem entre si, a $5 \%$ de probabilidade, pelo teste de Tukey. 
Outro ponto foi à capacidade do pulverizador automatizado em posicionar o diafragma para maior vazão de ar possível nas condições meteorológicas de menor capacidade evaporativa do ar, onde um maior fracionamento é desejado. Ao longo de uma jornada diária de trabalho, o sistema permitiu um ajuste fino dos parâmetros técnicos da pulverização e permite-se assim reduzir as perdas por evaporação conforme constatado por Balanet al. (2008) e Nascimento et al. (2012).

\section{CONCLUSÕES}

O diafragma aliado ao sistema eletrônico possibilitou a automatização do pulverizador pneumático, quanto à vazão de ar do ventilador. O pulverizador pneumático automatizado respondeu às diferentes condições meteorológicas. $\mathrm{O}$ tempo de resposta do sistema variou de 66,3 a 39,3 segundos.A automatização possibilitou a obtenção da vazão de ar, com uma interpretação simples do sistema através da ação dos LEDs.

\section{REFERÊNCIAS BIBLIOGRÁFICAS}

ALVARENGA, C.B.; TEIXEIRA, M.M.; CECON, P.R.; SIQUEIRA, D.L.; SASAKI, R.S.; RODRIGUES, D.E. 2013. Déficit de pressão de vapor d'água no ar na distribuição de líquido utilizando um pulverizador hidropneumático. Revista de Ciências Agrárias, Belém, v. 56, n.2, p.81-87.

ALVARENGA, C.B.; TEIXEIRA, M.M.; ZOLNIER, S.; SASAKI, R.S.; RINALDI, P.C.N. 2013. Controle automático do espectro de gotas de pulverizador hidro-pneumático em função do déficit de pressão de vapor d'água no ar. Pesquisa Agropecuária Tropical, Goiânia, v.43, n.1, p.26-33.

BALAN, M.G.; ABI-SAAB, O.J.G.; SILVA, C.G.; RIO, A. 2008. Deposição da calda pulverizada por três pontas de pulverização sob diferentes condições meteorológicas. Semina: Ciências Agrárias, Londrina, v.29, n.2, p.293298.

BALSARI, P.; MARUCCO, P.; TAMAGNONE, M. 2009. A crop identification system (CIS) to optimize pesticide applications in orchards. Journal of Horticultural Science \& Biotechnology, Dundee, ISAFRUIT Special Issue, 113-116.

BATTE, M. T.; EHSANI, M. R. 2006. The economics of precision guidance with auto-boom control for farmer-owned agricultural sprayers. Computers and Eletronics in Agriculture, Amsterdam, v. 53, 28-44.

DEBORTOLI, M.P.; TORMEN, N.R.; BALARDIN, R.S.; FAVERA, D.D.; STEFANELLO, M.T.; PINTO, F.F.; UEBEL, J.D. 2012. Espectro de gotas de pulverização e controle da ferrugemasiática-da-soja em cultivares com diferentes arquiteturas de planta. Pesquisa agropecuária brasileira, Brasília, v.47, n.7, p. 920-927.

DORUCHOWSKI, G.; SWIECHOWSKI, R.; HOLOWNICKI, R.; GODYN, A. 2009. Environmentally-dependent application system (EDAS) for safer spray application in fruit growing. Journal of Horticultural Science \& Biotechnology, Dundee, ISAFRUIT Special Issue, 107-112, 2009. 
MANHANI, G.G.; TEIXEIRA, M.M.; FERNANDES, H.C.; ZOLNIER, S.; SASAKI, R.S. 2013. Developing a system to control the air flow a pneumatic sprayer. Bioscience Journal, Uberlândia, v.29, n.3.

NASCIMENTO, A.B.; OIVEIRA, G.M.; BALAN, M.G.; HIGASHIARA, L.R.; ABI SAAB, O.J.G. 2012. Deposição de glifosato e utilização de adjuvantes para diferentes pontas de pulverização e horário de aplicação. Revista Brasileira de Tecnologia Aplicada nas Ciências Agrárias, Guarapuava, v.5, n.2, p.105116.

RUEDELL, J. 2002. Tecnologia de aplicação de defensivos. Plantio Direto, Brasília, v.19, n.6, p.9-11.

SCHULLER, J.K. 1991. Design for dynamic response of sprayer-applicators. Journal of Fertilizer Issues, Manchester, v.8, n.3, p.69-73.

SILVA, K.O.; MORAES, S.O.; MIRANDA, J.H.; PALMIERI, A.M. 2007. Sistema automatizado para aquisição de dados de umidade relativa do ar. Engenharia Agrícola, Jaboticabal, v.27, n.3, p.630638.

VILLALBA, J.; HETZ, E. Deriva de productos agroquímicos - Efecto de las condiciones ambientales. In Tecnología de aplicación de agroquímicos. Argentina: Área de comunicaciones del INTA Alto Valle, 2010. cap. 3, p. 45-54.

YU, Y.; ZHU, H.; FRANTZ, J.M.; REDING, M.E.; CHAN, K.C.; OZKAN, H.E. 2009. Evaporation and coverage area of pesticide droplets on hairy and waxy leaves. Biosystems Engineering, Columbus, v. 104, n. 3, p. 324-334.

YU, Y.; ZHU, H.; OZKAN, H.E.; DERKSEN, R.C.; KRAUSE, C.R. 2009b. Evaporation and deposition coverage area of droplets containing insecticides and sprays additives on hydrophilic, hydrophobic, and crabapple leaf surfaces. Transactions of the ASABE, St. Joseph, v. 52, n.1, p.39-49.

Recebido em: 19\12〈2013 Aceito para publicação em: 26\11 2014 